\title{
Systems Design of a High Resolution Retinal Prosthesis
}

\author{
James D. Weiland ${ }^{1}$, Wolfgang Fink ${ }^{2,1}$, Mark S. Humayun ${ }^{1}$, Wentai Liu ${ }^{3}$,Wen Li ${ }^{4}$, Mohanasankar \\ Sivaprakasam $^{3}$, Yu-Chong Tai ${ }^{4}$, Mark A. Tarbell ${ }^{2}$ \\ ${ }^{1}$ Doheny Eye Institute, Department of Ophthalmology, Keck School of Medicine, University of Southern California, \\ Los Angeles, CA \\ ${ }^{2}$ Division of Physics, Mathematics and Astronomy, California Institute of Technology, Pasadena, CA, USA \\ ${ }^{3}$ Department of Electrical Engineering, University of California, Santa Cruz, CA, USA \\ ${ }^{4}$ Electrical Engineering, Division of Engineering and Applied Science, California Institute of Technology, \\ Pasadena, CA, USA
}

\begin{abstract}
Simulations of artificial vision suggest that 1000 electrodes may be required to restore vision to individuals with diseases of the outer retina. In order to achieve such an implant, new technology is needed, since the state-of-the-art implantable neural stimulator has at most 22 contacts with neural tissue. Considerable progress has been made towards that goal with the development of image processing, microelectronics, and polymer based MEMS. An image processing system has been realized that is capable of real-time implementation of image decimation and filtering (for example, edge detection). Application specific integrated circuits (ASICs) have been designed and tested to demonstrate closed loop power control and efficient microstimulation. A novel packaging process has been developed that is capable of simultaneously forming communication coils, interconnects, and stimulating electrodes.
\end{abstract}

\section{Introduction}

Several incurable eye diseases result in blindness for 100,000 's of individuals each year.[1] One proposed treatment for these conditions is a retinal prosthesis that will stimulate the retina at many distinct locations to create a pattern of neural activation and thus a visual perception.[2,3] In order to function properly, a retinal prosthesis will require the presence of cells in the retina. Therefore, diseases primarily limited to the outer retina are potentially treatable with a retinal prosthesis. The two most common outer retinal degenerative diseases are age-related macular degeneration (AMD) and retinitis pigmentosa (RP). AMD is more prevalent but RP is more severe. Electrical stimulation in human test subjects with these conditions, has demonstrated the feasibility of an electronic retinal prosthesis as a means of providing some degree of vision.[4] This paper describes progress towards a high-resolution retinal prosthesis designed to restore visual functions such as face recognition, mobility, and navigation.

\section{Background}

To design a high resolution retinal prosthesis, it is important to consider the system requirements with respect to the diseased tissue, the number of pixels needed for the patient to have useful vision, and the output requirements to elicit the perception of light with an electrode on the retinal surface.
Post-mortem evaluations of retinae with RP or AMD have shown that, in spite of photoreceptor degeneration, significant numbers of inner retinal cells remain. However, the neural circuitry is altered compared to normal retina. Psychophysical tests with the first prototypes show that phosphenes (artificially created sensation of light) can be reliably elicited in humans with severe RP. [5,6] Simulations of prosthetic vision with a retinal implant have predicted the number of electrodes needed to perform certain visual tasks. A number of studies have been performed that assess the visual task performance of normally sighted individuals while immersed in a simulated environment. These studies have been recently reviewed.[7] The combined results suggest that a retinal prosthesis must have 600-1000 individual pixels to restore function such as face recognition, reading, and unaided mobility.

\section{Systems Design}

The system is designed to restore a functional level of vision to blind individuals so it will have 1000 individual electrodes. Each electrode will have a dedicated current driver. While demultiplexing is possible, to reduce the number of current drivers, it is not yet known if stimulus pulses should be simultaneous or interleaved, so maximum flexibility should be maintained at this development stage.

Systems power and data are delivered inductively via a dual band telemetry system. The receiver coils are positioned in the front half of the eye, an electronics module in the vitreous cavity, and an electrode array on the epiretinal surface at the back of the eye. The electronics module has ASICs and passive components for AC/DC power conversion, data demodulation, and stimulus current generation based on received data. The implant coils, interconnects, and electrodes are formed during a single parylene micromachining process described below. The implanted system is controlled by an external system, which includes a camera for image acquisition, image processing hardware, a power amplifier and data modulation.

Our progress to date supports the feasibility of the overall system (Fig. 1). We have demonstrated real-time image processing, dual-band telemetry, high-voltage circuit 
drivers, and parylene substrate electrodes, interconnects, and coils. We have also performed biological experiments to assess the feasibility of the implantation of this device.

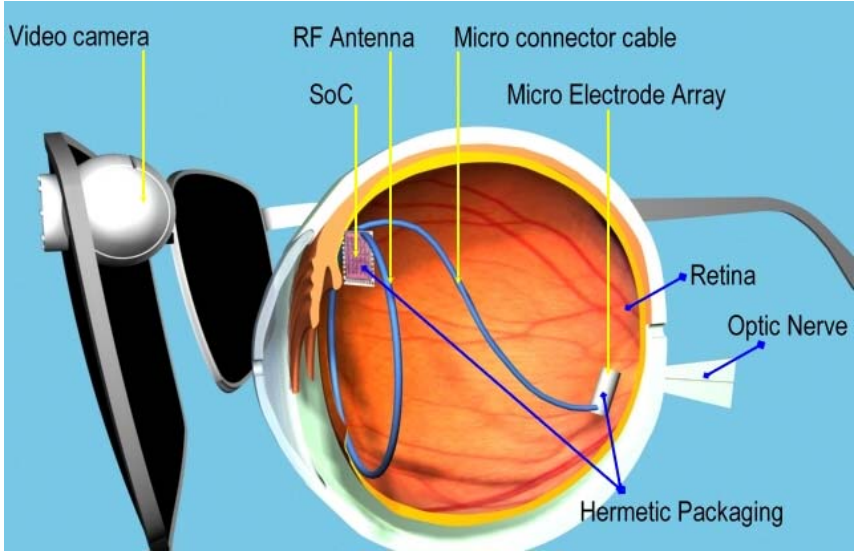

Fig. 1 - Conceptual diagram of retinal prosthesis system. A video camera on a pair of glasses captures information. A wearable computer (not shown) processes video information and transmits it to the implant. Power is also sent wirelessly via inductive coils. The implanted electronics decode the information and produces DC chip power from the induced AC voltage on the power coil. The implanted chip produces a pattern of stimulation based on the received data. The stimulus pulse pattern is applied to the retina via a microelectrode array.

\section{Real Time Image Processing - AVS}

To enhance and optimize the visual perception of retinal implant patients, we have created a comprehensive software package: the Artificial Vision Simulator (AVS) [8, 9]. AVS interfaces with standard video cameras and presents the captured video stream in a user-defined pixelation. AVS employs standard image processing algorithms (e.g., $[10,11]$ ), as well as newly developed, customized image manipulation algorithms, such as gray scale and contrast enhancement, to modify the captured video stream (Fig. 2). All algorithms are implemented to be executable in real time (30 fps) and can be engaged in arbitrary, user-defined order. These features afford an unprecedented flexibility in manipulating the video stream of the external camera in real time for the benefit of retinal implant patients, both by a technician when fitting the system to the patients or, within limits, by the patients themselves. We have begun migrating the currently laptop-based AVS processing system onto a portable, battery-powered micro-processor platform for enhanced user mobility.

\section{Power and Data Telemetry}

In order to achieve high power efficiency and large data rate, the system has dual-band architecture with two pairs of coils to transmit power and data with two separate carriers (Fig. 3). This allows independent optimization of the power and data telemetry links. The data clock and power clock are synchronized at the transmitter side and received at the implant side. The dual band telemetry system has been validated for fully wireless recovery of power, data, and system clock at the implant side.
A reverse data telemetry link transmits implant power information using load shift keying to the external unit that uses the information to transmit the 'just-needed' power to increase efficiency and battery life [12]. The forward data telemetry employs differential phase shift keying (DPSK), where ' 1 ' is coded as a phase shift of $180^{\circ}$ and ' 0 ' is coded as zero phase shift. The data signal is modulated on a $20 \mathrm{MHz}$ carrier at a data rate up to $2 \mathrm{Mbps}$. The demodulation uses subsampling and analog demodulation to recover data at the implant that was verified through a custom integrated circuit [13].
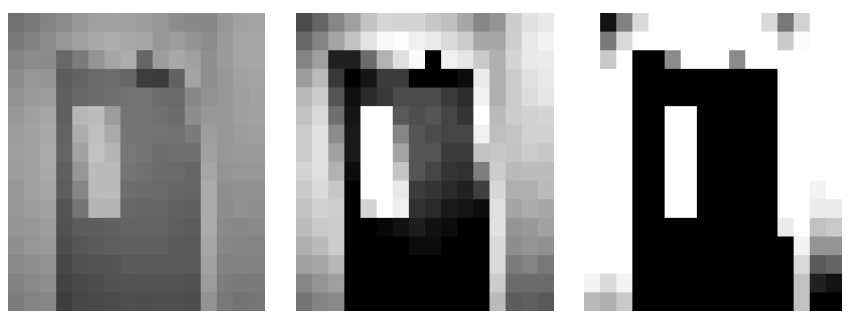

Fig. 2 - Artificial Vision Simulator (AVS) [8, 9]: user-defined pixelation and real time image processing of captured camera video stream, (resolution here: $16 \times 16$ ): raw camera image of door (left), gray scale enhancement (middle), and additional contrast enhancement (right).

To integrate the Artificial Vision Simulator with the high resolution retinal prosthesis system, an extensible Ethernetbased (i.e., TCP/IP) Data Exchange Protocol has been developed to facilitate the transfer of the AVS-manipulated (video) output data to the Power \& Data Telemetry system. This Ethernet-based protocol allows for maximum flexibility between systems, and is capable of two-way data transfer as well as status message transmission. Contingency measures are integrated into the protocol, providing for negative acknowledgements and data resend requests, should the need arise in which data is required to be resent. Fig. 3 shows demonstration data from the dual band telemetry systems, where AVS is used to provide data to the telemetry system and a MEMS coil (described below) is used to receive data.

\section{Stimulator Microelectronics}

The semiconductor process for the stimulator design is mainly governed by the stimulation voltage at the electrode. Based on biological data, we estimate the maximum stimulation compliance voltage to be $9 \mathrm{~V}$. Given the biphasic current pulses and monopolar mode of stimulation, the microelectronics should account for twice the stimulation voltage in addition to overhead incurred by the stimulation circuit, power recovery and regulation. This requires a high voltage semiconductor process rated for up to $30 \mathrm{~V}$. The choice of process is also dictated by the need for small feature size of transistors to achieve a highly miniaturized chip. This is against the general trend of semiconductor fabrication processes where reduction of feature size is accompanied by reduction of operating voltage. 
To accommodate the special requirements of high voltage and high density microstimulator, a high voltage deep sub micron CMOS process is employed. This enables a high density microstimulator by taking advantage of small feature size for digital circuitry and still achieving high voltage compliance [14]. Because this particular process also provides low voltage transistors, the digital controller is integrated with high voltage current drivers and power regulators on a single chip. A high density microstimulator consisting of power receiver, forward data receiver, reverse data transmitter and stimulation, analog driver and digital controller is currently under fabrication.

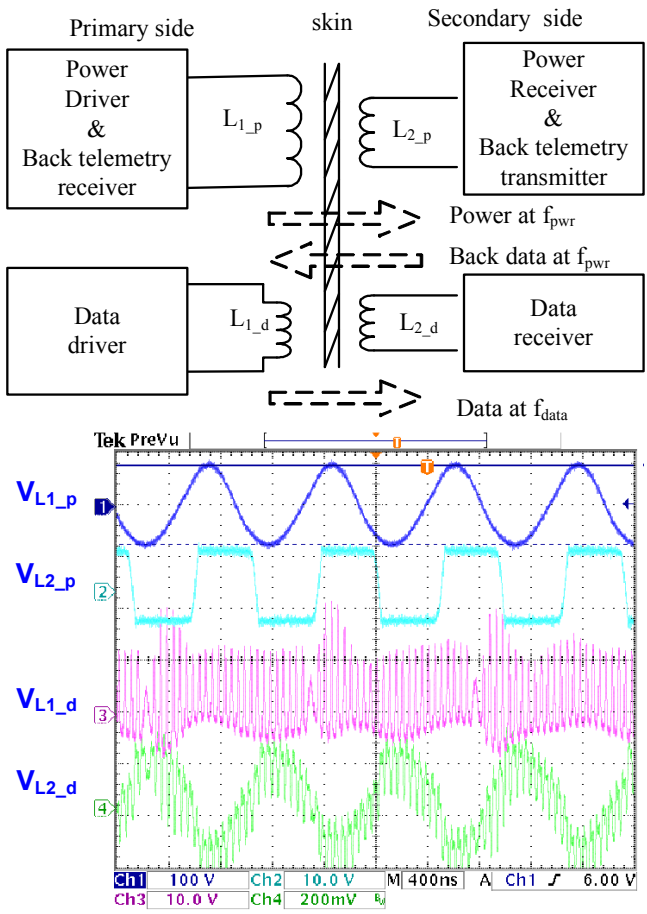

Fig. 3 - (Top) Dual band telemetry block diagram (Bottom) Demonstration of simultaneous power and data transmission. Note a MEMS coil is used for data receive.

\section{Packaging, Coil, Interconnects, and Electrodes}

We have developed a chip-level integrated interconnect (CLI2) packaging technology to assemble foundry-fabricated chips with RF coils, multi-electrode array and other external components, and have now extended it to incorporate silicon housings and functional parylene-based devices. Prefabricated chips and passive components can be embedded in a carrier silicon wafer and sealed with parylene. Subsequent fabrication of other surface MEMS components, such as electrode arrays and coils, can be performed on the same substrate using a parylene-based skin technology. Fig. 4 shows the detailed process flow, which starts with a standard 4 inch wafer coated with a layer of sacrificial photoresist. A $5 \mu \mathrm{m}$ layer of parylene $\mathrm{C}$ is deposited on top of the photoresist. To secure parylene on the substrate, parylene anchors surrounding the chip housings are etched into the substrate using deep reactive-ion etching (DRIE).
After parylene deposition, a $500 \mathrm{~nm}$ layer of gold is deposited using an E-beam evaporator, and patterned to form the first layer of coil wires. Next, cavities matching the chip dimensions are etched into the parylene and silicon using oxygen plasma reactive-ion etching (RIE) and Bosch process, and the chips are then dropped into the cavities to self-align with lateral displacements less than $10 \mu \mathrm{m}$. Epoxy is applied to the bottom of the cavities as needed in order to compensate for cavity depth inaccuracy and to fill the gaps surrounding the chips. Parylene deposition is performed again to seal the chips and to form an insulation layer between two metal layers, followed by oxygen plasma etching to properly open interconnection vias. A second $500 \mathrm{~nm}$ layer of gold is then deposited and patterned to form the top layer of coil wires and interconnects. Another $5 \mu \mathrm{m}$ layer of parylene $\mathrm{C}$ is coated on top and patterned using oxygen plasma in the RIE to define the profile of devices. Through-wafer trenches surrounding the chips are then etched from the backside using Bosch process, and the flexible skin is released from the substrate, carrying the chips encapsulated in silicon housings. Lastly, the entire device can be sealed with parylene as a protection layer. (a)

(b)

(c)

(d)

(e)

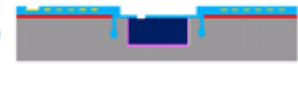

(f)

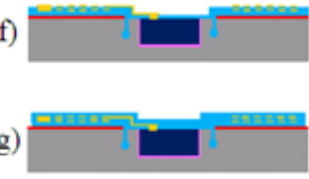

(h)

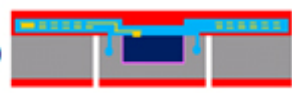

(i)

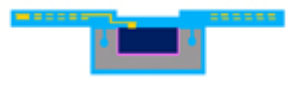

Fig. 4 - Process flow for CL-I2

A parylene electrode array has been implanted in an animal model to demonstrate its biocompatibility.[15] Fig. 5 shows an array of 1000 individual electrodes on the surface of the retina (as photographed through a dilated pupil). The implants were retrieved after 6 months. Examination of the retina showed only minimal response to the implant, well within acceptable limits.

The coil design is highly dependent on the application. Data telemetry and low-power system can use thin-film metal coils, but the power requirements for a retinal prosthesis $(10-100 \mathrm{~mW})$ will require a high quality, low resistance coil. Progress towards microfabrication of coils within the overall packaging process is demonstrated through integration of an EM4100 read-only RFID chip (Fig. 6). A square planar coil is designed for wireless power and data transmission. The coil consists of two metal layers with 22 turns on each layer (Fig. 7). The overall coil size is 
$2 \mathrm{~cm}$ by $2 \mathrm{~cm}$. Due to the low power consumption of the wireless chip, only the coil is needed to power the circuit, and no extra capacitor is required. An RFID reader module verified communication to the chip via the microfabricated coil (Fig. 8). For applications that require higher power and greater operating ranges, the power transfer efficiency can be enhanced by increasing the number of turns, the metal thickness, or the metal layers.

Accelerated-lifetime testing has been performed to evaluate the durability of the parylene packaging. RFID chips and coils are separately coated with $10 \mu \mathrm{m}$ of parylene-C, and then annealed at $200{ }^{\circ} \mathrm{C}$ for 2 days in a vacuum oven. Samples are tested in hot saline under different conditions, and their failure mechanisms are monitored at distinct time intervals using both optical and electron-beam metrologies. For the parylene encapsulated chips, unpowered testing is performed at $77^{\circ} \mathrm{C}$, and the chips still function well after 90 days. Parylene coated coils are tested under both passive and active electrical stressing at two different temperatures, and their MTTF at body temperature is extrapolated using an Arrhenius model. The preliminary results estimate that parylene packaging can remain intact at $37{ }^{\circ} \mathrm{C}$ for over 60 years, which is very promising for biomedical implantations.

\section{Conclusions}

Image processing, telemetry, and microfabrication technology is being advanced to support a 1000-channel retinal prosthesis. Integration of a final system is the next step in development.

\section{Acknowledgment}

This material is based on work supported by the National Science Foundation under Grant No. EEC-0310723.

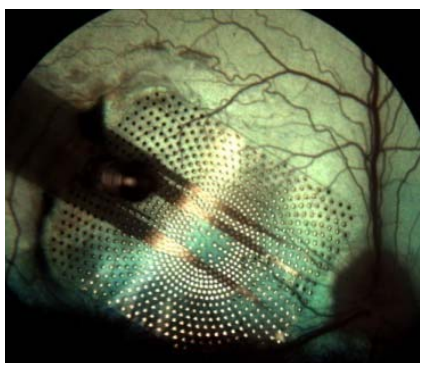

Fig. 5 - 1000 electrode, parylene substrate array implanted on retinal surface. Electrodes covers $5 \times 6 \mathrm{~mm}$ area.

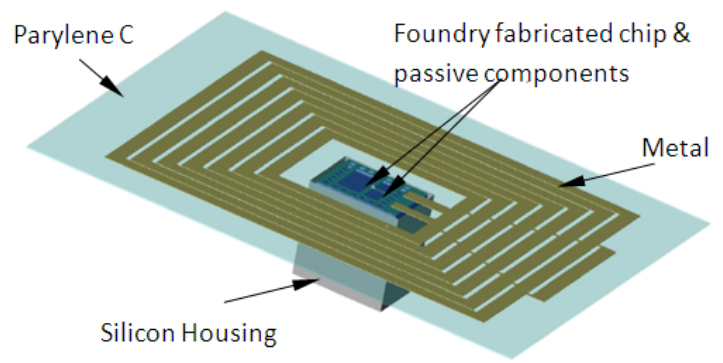

Fig. 6 - Diagram of chip with coil packaged using CL-I2 technology

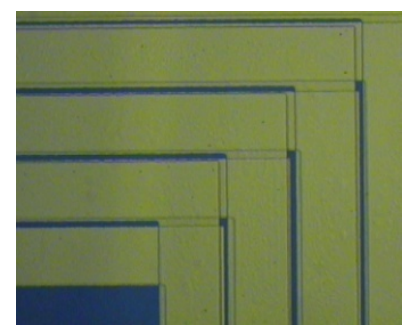

Fig. 7 - Close of MEMS coil, showing two overlapping metal layers, separated by parylene insulating layer

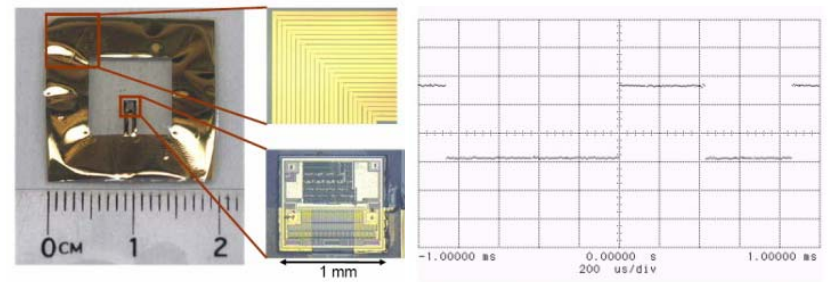

Fig. 8 - Package and Coil testing used an RFID chip. (left) RFID with MEMS coil. (right) Test data from RFID reader.

\section{References}

1. E Margalit and S Sadda. Retinal and Optic Nerve Diseases, Artif. Organs, 2003 Nov;27(11):963-74

2. E Zrenner, Will retinal implants restore vision? Science, 2002 295(5557): $1022-5$

3. J. Weiland, W. Liu, M. Humayun. Retinal Prosthesis. Annu Rev Biomed Eng. 2005;7:361-401

4. D. Yanai, J. Weiland, M. Mahadevappa, et. al. Visual performance using a retinal prosthesis in three subjects with retinitis pigmentosa. Am J Ophthalmol. 2007 May;143(5):820-827.

5. J.F. Rizzo, J. Wyatt, J. Loewenstein, S. Kelly, D. Shire. Methods and perceptual thresholds for short-term electrical stimulation of human retina with microelectrode arrays. Invest Ophthalmol Vis Sci. 2003;44(12):5355-61.

6. C. de Balthasar, S. Patel, A. Roy et.al. Factors affecting perceptual thresholds in epiretinal prostheses. Invest Ophthalmol Vis Sci. 2008 Jun;49(6):2303-14.

7. G. Dagnelie. Psychophysical Evaluation for Visual Prosthesis. Annu Rev Biomed Eng. 2008

8. Fink W, Tarbell M (2005) Artificial Vision Simulator (AVS) for Enhancing and Optimizing Visual Perception of Retinal Implant Carriers, Invest. Ophthalmol. Vis. Sci. 2005 46: E-Abstract 1145

9. Liu W, Fink W, Tarbell M, Sivaprakasam M (2005) Image Processing and Interface for Retinal Visual Prostheses; ISCAS 2005 Conference Proceedings (invited), Kobe, Japan, 2927- 2930 Vol. 3

10. Russ JC, "The Image Processing Handbook," CRC Press, 2002

11. Myler HR, and Weeks AR, "The Pocket Handbook of Image Processing Algorithms in C," Prentice Hall PTR, 1993

12. G. Wang, W. Liu, M. Sivaprakasam, and G. A. Kendir, "Design and Analysis of an Adaptive Transcutaneous Power Telemetry for Biomedical Implants," IEEE Transactions on Circuits and Systems I, Volume: 52, Pages: 2109 - 2117, October 2005.

13. M. Zhou, W. Liu, "A Non-Coherent PSK Receiver with Interference-canceling for Transcutaneous Neural Implants," ISSCC Digest of Technical Papers, pp. 156 - 157, Feb 2007

14. J. Kim, M. Chae, M. Sivaprakasam, and W. Liu, "An Integrated High Voltage Stimulator for High Density Neural Interfaces,” BMES Annual Meeting, September 2008

15. D.C. Rodger, A.J. Fong, W. Li, H. Ameri, I. Lavrov, H. Zhong, S. Saati, P. Menon, E. Meng, J.W. Burdick, R.R. Roy, V.R. Edgerton, J.D. Weiland, M.S. Humayun, and Y.C. Tai. High-density flexible parylene-based multielectrode arrays for retinal and spinal cord stimulation. TRANSDUCERS 2007: 1385-1388. 